\title{
Immediate Vicryl mesh insertion after lumpectomy, advantage overview
}

\author{
Abdullah Salem Al Haider, Mana Ali mueidh Al hajlan, Abdulaziz Abdullah Alhazmi, Yaqoub \\ Mubarak Ali Alhamami, Nasser Naji Mohsen Al harthi, Mohammed Hundur Alasmari, \\ Alhussain Abdullah Jarullah Albudaydi
}

Najran University, Medical Collage

\begin{abstract}
Background: oncoplastic surgical treatment is defined as mix of excision of a tumor, with proper margin consisting of lumpectomy or mastectomy and prompt reconstruction of the bust, this may require applying mesh to stabilize the structure beneath the skin. Objective: we discussed the efficiency of Vicryl mesh and it usage in surgery after lumpectomy. Method: we searched in detailed following electronic databases; (PubMed, Embase, Google scholar) for relevant studies published in English language with human subject only up to July,2018. Results: absorbable surgical mesh is the most current method in oncoplastic surgery. The simpleness of the time-preserving method that is comfortably appropriate has actually been reported to have substantial benefits for specialists in addition to a fairly affordable and acceptable aesthetic end results. Conclusion: elimination of just the area of cancer by lumpectomy intends to keep most of the bust tissue, is much less of a modification to your body compared to mastectomy and has a healing time of a couple of weeks. Immediate Vicryl mesh insertion is a simple approach. Additionally, surgeons ought to think about mesh use when constructing patients who are mosting likely to or have actually obtained radiotherapy to the bust; vicryl mesh is a reliable choice to acellular dermal matrix in non-irradiated bust reconstruction and is accessible at approximately one twentieth the expenses. Acellular dermal matrix or complete muscular tissue coverage could be preferable in irradiated reconstructions.
\end{abstract}

Keywords: Vicryl mesh, insertion, advantage, lumpectomy, area of cancer.

\section{Introduction:}

Breast cancer is one of the most diagnosed cancer in women worldwide, with almost 1.7 million new situations identified in $2012^{[1]}$. Breast cancer happens when unusual cells in the breast multiply uncontrollably to create a tumor. While , most cases of breast cancer happen in females it does happen in men too, although this is rare (concerning 1\% of instances) ${ }^{[1]}$. Oncoplastic surgical treatment is defined as mix of excision of a tumor, with proper margin consisting of lumpectomy or mastectomy and prompt reconstruction of the bust ${ }^{[2]}$. This method has really ended up being promptly a lot more accepted throughout Western countries, permitting the achievement of oncologically secure margin and also enough aesthetic results ${ }^{[3]}$. Lately, similar results have actually been reported for local reappearance as well as survival result ${ }^{[2,3]}$. Absorbable surgical mesh is the most current method in oncoplastic surgery. The simpleness of the time-preserving method that is comfortably appropriate has actually been reported to have substantial benefits for specialists in addition to a fairly affordable and acceptable aesthetic end result ${ }^{[2]}$. Oncoplastic surgical treatment and breast reconstruction are crucial parts of the therapy of breast cancer. Acellular dermal matrices (ADMs) have been utilized for the reconstruction of the lower pole as a result of numerous advantages; however, its cost is considered as a major disadvantage in this era of concern for the allocation of health-care funds. Recently, polyglactin 910 (Vicryl) mesh has been released as an alternative. We discussed the efficiency of Vicryl mesh and it usage in surgery after lumpectomy.

\section{Methodology:}

We searched in detailed following electronic databases; (PubMed, Embase, Google scholar) for relevant studies published in English language with human subject only up to July 2018. Our search method performed using mesh terms as breast cancer lumpectomy reconstruction breast surgery with absorbable mesh insertion (Vicryl mesh).

\section{Discussion}

- What is a lumpectomy?

Lumpectomy is a surgical procedure that entails eliminating a presumed malignant (malignant) tumor or various other uncommon tissues as well as a small portion of the surrounding bust 
tissue ${ }^{[2]}$. This tissue is then tested to determine if it contains malignant cells. A number of lymph nodes may additionally be removed to check them for malignant cells (sentinel lymph node biopsy or axillary breakdown). If malignant cells are found in the tissue example or nodes added surgical procedure or treatment may be essential. Women who go through a lumpectomy generally obtain radiation therapy (RT) for about 6 weeks complying with the procedure to kill any type of cancer cells that may have been missed with the removal of the tumor. Lumpectomy is also described as partial mastectomy, wedge resection, breast-sparing treatment, breast-conserving therapy, breastconserving surgical treatment, excision biopsy, tylectomy, segmental excision and quadrantectomy ${ }^{[2,3]}$. Lumpectomy is applied to treat both invasive cancers cells of the bust (invasive ductal carcinoma or invasive lobular carcinoma) as well as ductal carcinoma in situ (DCIS).A couple of years earlier, the typical bust cancer surgical procedure was radical mastectomy, which involves the complete elimination of the bust, muscle mass from the breast wall and all the lymph nodes in the underarm. Breast-conserving surgical procedure has actually changed extreme mastectomy as the preferred surgical therapy because lumpectomy is developed to leave the natural appearance as well as aesthetic quality of the breast mostly undamaged while getting rid of the malignancy and also a little margin of regular bust tissue. Furthermore, researches have revealed that lumpectomy with radiation therapy is as reliable as mastectomy in treating breast cancer ${ }^{[4]}$. The location and size of the lump determines how much of the breast is removed during a lumpectomy. A quadrantectomy, as an example, includes eliminating a quarter of the breast. Prior to surgical treatment, a woman should go over with her doctor what amount of the bust will certainly be entailed to ensure that she can understand just what to expect ${ }^{[4]}$.

The size of the cancer in connection with the size of the bust is the major aspect that a lady's doctor takes into consideration to establish if a lumpectomy is a suitable therapy. Furthermore, several of the functions of the cancer (if it is constrained to one location of the bust and also does not involve the skin or breast wall) aid the doctor establish if lumpectomy is appropriate. A lot of ladies who are diagnosed with breast cancer, especially those who are detected early, are taken into consideration good prospects for lumpectomy [5]. However, under some scenarios, lumpectomy is not a suggested surgery for some ladies. These variables consist of the following ${ }^{[4,5]}$.

- Multiple cancers cells in separate areas of the very same breast: this indicated that the possibly malignant tissue could not all be removed from a solitary area, suggesting that the breast might become considerably injured as a result of lumpectomy.

- Prior lumpectomy with radiation women who have had a lumpectomy with radiation treatment to remove cancer cannot have more radiation; as a result, they normally require a mastectomy if they experience cancer once again in the exact same bust.

- Extensive cancer: since a lumpectomy eliminates a certain location with hatred, this cancer therapy option would certainly be improper if the cancer has actually spread to various other areas.

- Problematic tumors: a tumor that is rapidly expanding or has connected itself to a neighboring framework, such as the chest wall or skin, might call for surgical treatment that is a lot more extensive to remove the tumor.

- Pregnancy: radiation therapy, which generally complies with the lumpectomy, could harm the female's fetus.

- Large tumors: lumpectomy to remove a tumor that is bigger than 5 centimeters in diameter might considerably damage the bust. However, in some cases, the dimension of the tumor could be able to be decreased with radiation treatment or endocrine treatment, to a dimension that is more manageable with lumpectomy. Little busts, specifically those which contain huge lumps, might also be substantially disfigured after lumpectomy.

- Preexisting conditions that make radiation treatment more high-risk than normal: Radiation treatment could mark or damage connective tissue in women with collagen vascular conditions, such as scleroderma or lupus erythematosus.

Prior radiation to the chest area, as an example, to deal with Hodgkin's condition.

Some ladies may favor the mastectomy to lumpectomy in order to really feel even more certain that they will not develop bust cancer once more. Other women might not really feel 
comfy with radiation treatment or have the ability to commit to a set of radiation treatments, which might entail an undesirable time dedication or extensive travel. In a lot of situations, though, ladies could securely choose in between lumpectomy and mastectomy.

- What are the risks associated with a lumpectomy?

The dangers entailed with lumpectomy are typical of any kind of operation. These include infection, bleeding, and damage to close-by cells. General anesthesia is additionally connected with some threat, although rare. Few women that are usually healthy experience a severe problem from general anesthesia.

The complying with additional medical and aesthetic risks related to lumpectomy ${ }^{[2-5]}$ :

- Depending on how much of the bust was gotten rid of during the lumpectomy, the appearance of the bust could modify. The bust may have a visible scar or dimpling as well as might become asymmetrical in connection with the other bust.

- Some females could experience some nerve damages, which could trigger a loss of sensation in the breast. Some females who obtain a guard node biopsy or axillary lymph node breakdown could shed some sensation in the underarm or variety of motion in the shoulder. Various other feelings could also be impacted.

- Two to ten percent of women who undergo axillary lymph node dissection might develop lymphedema, which is the build-up of liquid in the location of the lymph nodes. This problem can establish right away or perhaps years after the treatment. If females have actually had an axillary lymph node dissection, they must avoid anything limited around the arm (like a tourniquet used to draw blood, a blood pressure cuff, or a tight band on a sleeve) on that particular side for the rest of their life. Phlebitis may develop, which means the arm vein becomes inflamed.

- Cellulitis may occur. This is inflammation of the skin of the arm.

Risks associated with radiation therapy include the following:

- Fatigue
- Nausea

- Hair loss of the treated area

- Loss of appetite

- Skin reactions of the treated area, including redness or rash

- Vicryl mesh insertion

Bust reconstruction makes a variety of demands on the plastic surgeon: acceptable size, balance, and form; practical tissue quality; reasonable period to completion; long-term stability; and also, we wish, a reduced rate of morbidity and also discomfort. The numerous a cellular dermal matrix items- mainly, Alloderm- have gained a place as beneficial adjuncts in reconstructing the bust. Till acellular dermal matrix became available, sub muscular placement was the criterion standard for expander-based reconstruction, with some specialists making sensible use of dual-plane placement ${ }^{[6]}$ Acellular dermal matrix holds the guarantee of better interpretation of the inframammary layer, greater first fill volumes, potentially much less discomfort, and a shorter time program of inflation. Acellular dermal matrix incorporates right into the host tissue and also retains strength, so the pectoralis will certainly not displace throughout expansion. Using bigger pieces of acellular facial matrix necessarily causes much less of the expander being covered by the pectoralis, and also similarly limits the putative benefit of having the expander (and succeeding implant) covered by muscular tissue. Although it has been well shown that acellular dermal matrix will vascularize with time, ${ }^{[7]}$ it is difficult to believe that the blood supply within the acellular facial matrix will ever before approach that of native muscle mass.

Vicryl mesh has been used in numerous areas of surgery, consisting of released reports as gynecology ${ }^{[8]}$, whole surgery ${ }^{[9]}$ and also neurosurgery ${ }^{[10]}$. Certainly, we picture most cosmetic surgeons have actually utilized it eventually in their occupation. Ethicon does not release a strength dissolution curve for Vicryl mesh- if we assume its properties resemble those of Vicryl suture, the mesh ought to retain about 50 percent of its original stamina at 3 weeks and dissolve totally in 8 to 10 weeks. In the research study of Haynes et al. ${ }^{[11]}$ they used Vicryl mesh in comparable fashion to acellular dermal matrix, and to serve the exact same first function- securing the pectoralis muscle in the preferred setting during the beginning of healing and also expansion, and helping to 
prevent lateral migration of the expander. With time, the muscular tissue heals to the substandard mastectomy skin flap and capsule starts to create around the expander, keeping muscle mass position as the vicryl mesh eventually loses tensile strength and liquifies. They thought that this adherence to the overlapping soft tissue safeguards the muscle throughout the later stages of development. It may appear logical that as soon as the mesh dissolves, the pectoralis might change in a superomedial direction ${ }^{[11]}$. In the team of patients, when the mastectomy incision was reopened to eliminate the expander and also area the permanent implant, muscle constantly existed under the entire incision. This does not confirm that displacement did not occur, yet it did not strike a substantial level. In various other patients where they have utilized AlloDerm, especially in larger portions, it is not uncommon for muscle mass to underlie only the median part of the resumed incision, with vascularized AlloDerm covering the expander sideways ${ }^{[11]}$.Their average first expander fill quantity is less than that reported by numerous authors using acellular dermal matrix ${ }^{[6,12,13]}$. The conclusion from this that the inset of the mesh was on ordinary rather tighter, as well as the surface area of the implanted mesh fairly much less, than the ordinary record using acellular dermal matrix. Fundamentally, a higher fraction of the expander was originally covered by the pectoralis muscular tissue, as well as much less was covered by the Vicryl mesh. This tighter inset can understandably contribute to the reasonably reduced rate of seroma as well as probably clarify the previously pointed out finding of muscle covering a substantial section of the expander when exposed at the second-stage surgical procedure. Our postoperative problems slant greatly toward the little team of irradiated busts. In the non-irradiated breasts, there was one infection/ expander loss, one seroma, as well as 2 circumstances of mastectomy flap skin necrosis, both little enough for primary reclosure. Most of our issues were in the patients who received radiotherapy, whether prior to or after restoration. Acellular dermal matrix products likewise reveal greater difficulty rates in irradiated patients, with overall issue rates of 40 percent or greater, as reported by numerous writers ${ }^{[14-16]}$. Nguyen et al., in their contrast of 321 busts, 97 of which were irradiated, discovered a 10.7 percent explanation rate in irradiated busts rebuilded with AlloDerm, compared to a 0 percent explanation rate in those without AlloDerm ${ }^{[17]}$. There were only eight irradiated busts in our research, a small number where to draw conclusions; nevertheless, we would certainly recommend caution being used vicryl mesh in patients either formerly irradiated or for which postoperative irradiation is likely, unless the soft tissue coverage is especially robust.

The long-lasting follow-up averages 43 months, a better length compared to the majority of researches entailing AlloDerm. One patient developed a late infection 3 years after placement of the permanent implant, as well as one established a symptomatic contracture 4 years after implant positioning. It unlikely that the Vicryl mesh added to these late-stage troubles. The long-lasting revision rate in our non irradiated busts was 16.2 percent, above the 4 percent rate reported at 18 months by Spear yet less than the Core Study rates reported by Mentor (27 percent at 3 years) and Allergan (40.9 percent at 4 years) ${ }^{[18,19]}$. Of the three busts in the study where the patient chosen alteration surgical treatment for implant placement modification, 2 engaged medial/lateral modifications, which we feel were unassociated to using Vicryl mesh. The $3^{\text {rd }}$ showed a substandard malposition of the inframammary layer, and also although this was likely attributable to an extremely hostile release at the time of irreversible implant positioning, it is feasible this surgical treatment might have been prevented if acellular dermal matrix had been made use of along the inframammary layer at the preliminary treatment. There are speculative data that acellular dermal matrix might inhibit the development of pseudoepithelium around an implant, and this could represent evidence that acellular dermal matrix decreases the incidence of capsular contracture ${ }^{[21-23]}$ Rice $\boldsymbol{e t}$ $a l .{ }^{[20]}$ carried out a comparison of Vicryl mesh, AlloDerm and also Surgisis (Cook Medical, Bloomington, Ind.) in a rat hernia design, examining the strength of incorporation at baseline, 30 days, and also 60 days. Vicryl mesh had a reduced preliminary strength compared with AlloDerm (0.091 MPa versus $0.125 \mathrm{MPa}$, specifically) however a greater supreme toughness $(0.265 \mathrm{MPa}$ versus 0.23 $\mathrm{MPa}$, specifically) ${ }^{[20]}$. In appearing contrast to its greater strength, they also found much less collagen deposition in the repair services carried out with Vicryl mesh than those 
executed with AlloDerm. If lower quantities of collagen relate to a thinner capsule, this very same impact, if present in humans, can contribute to the reduced incidence of longlasting contracture seen in this research. At the health center where most of these instances were performed in Haynes et.al research, Vicryl mesh cost was $\$ 365.80$ for a portion 6 inches square (approximately $15 \times 15 \mathrm{~cm}$ ), as well as a $6 \times 12-\mathrm{cm}$ piece of AlloDerm is $\$ 2527.00^{[11]}$. When determined as cost each location, the disparity is a lot more noticable: $\$ 1.57$ each $\mathrm{cm} 2$ for Vicryl mesh compared to $\$ 35.10$ each $\mathrm{cm} 2$ for AlloDerm. When doing bilateral reconstructions, a solitary package of Vicryl mesh offers appropriate material for both sides.

\section{Conclusion}

Elimination of just the area of cancer by lumpectomy intends to keep most of the bust tissue, is much less of a modification to your body compared to mastectomy and has a healing time of a couple of weeks. Immediate Vicryl mesh insertion is a simple approach. Additionally, surgeons ought to think about MESH use when constructing patients who are mosting likely to or have actually obtained radiotherapy to the bust; Vicryl mesh is a reliable choice to acellular dermal matrix in nonirradiated bust reconstruction and is accessible at approximately one twentieth the expense. Acellular dermal matrix or complete muscular tissue coverage could be preferable in irradiated reconstructions. Lower-tocomparable occurrence of infection and also repair failing information when compared with ADM should motivate surgeons to research its use. Although the evidence is restricted, polyglactin 910 (Vicryl) mesh for IBR appears to be a potentially risk-free, reliable, as well as less expensive choice to ADM. Prospective studies are should more define its effectiveness. In an overloaded health-care system, assessing treatment choices and also minimizing prices end up being another function of the modern doctor; the pursuit of a cost-effective and also secure therapy comprises the $3 \mathrm{rd}$ and also final pillar of modern bust reconstruction.

\section{References:}

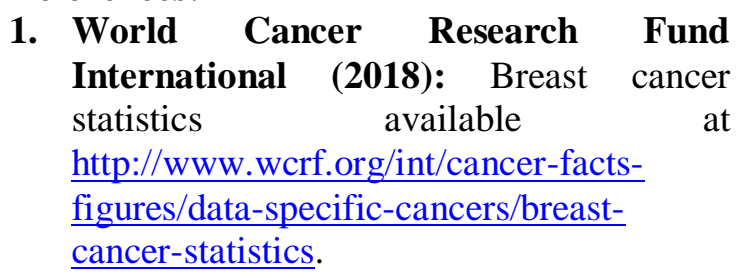

2. Koo MY, Lee SK, Hur SM et al. (2011): Results from over one year of follow-up for absorbable mesh insertion in partial mastectomy. Yonsei Medical Journal, 52(5):803-808.

3. Clough KB, Kaufman GJ, Nos C, Buccimazza I and Sarfati IM(2010): Improving breast cancer surgery: a classification and quadrant per quadrant atlas for oncoplastic surgery. Ann. Surg. Oncol.,17:1375-1391.

4. Fitoussi AD, Berry MG, Famà $F$ et al. (2010): Oncoplastic breast surgery for cancer: analysis of 540 consecutive cases . Plast. Reconstr. Surg.,125: 454-462.

5. Caruso F, Catanuto G, De Meo L et al. (2008): Outcomes of bilateral mammoplasty for early stage breast cancer. Eur. J. Surg. Oncol.,34:1143-1147.

6. Spear SL, Parikh PM, Reisin E et al. (2008): Acellular dermis-assisted breast reconstruction. Aesthetic Plast. Surg., 32:418-425.

7. Becker S, Saint-Cyr M, Wong C et al. (2009): AlloDerm versus DermaMatrix in immediate expander-based breast reconstruction: A preliminary comparison of complication profiles and material compliance. Plast. Reconstr. Surg., 123: 107-108.

8. Allahdin S, Glazener $\mathrm{C}$ and Bain $\mathrm{C}$ (2008): A randomised controlled trial evaluating the use of polyglactin mesh, polydioxanone and polyglactin sutures for pelvic organ prolapse surgery. J. Obstet. Gynaecol., 28:427-431.

9. Tobias AM and Low DW (2003): The use of a subfascial Vicryl mesh buttress to aid in the closure of massive ventral hernias following damage-control laparotomy. Plast. Reconstr. Surg.,112: 766-776.

10. Verheggen R, Schulte-Baumann WJ, Hahm G et al. (1997): A new technique of dural closure: Experience with a Vicryl mesh. Acta Neurochir. (Wien),139: 10741079.

11. Haynes DF and Kreithen JC (2014): Vicryl mesh in expander/implant breast reconstruction. Plastic and Reconstructive Surgery, 34(5):892-899.

12. Sbitany H, Sandeen SN, Amalfi AN et al. (2009): Acellular dermis-assisted prosthetic breast reconstruction versus complete submuscular coverage: a head-to- 
head comparison of outcomes. Plast. Reconstr. Surg., 124: 1735-1740.

13. Chun YS, Verma $K$, Rosen $H$ et al. (2010): Implant-based breast reconstruction using acellular dermal matrix and the risk of postoperative complications. Plast. Reconstr. Surg., 125:429-436.

14. Clemens MW and Kronowitz SJ (2012): Acellular dermal matrix in irradiated tissue expander/implant-based breast reconstruction: Evidence-based review. Plast. Reconstr .Surg., 130: 27-34.

15. Blount AL, Armstrong SD, Lineberry K et al. (2011): Complications associated with radiation in tissue expander breast reconstruction with human acellular dermal matrix. Plast. Reconstr. Surg., 127:15-22.

16. Weichman KE, Cemal Y, Albornoz CR et al. (2013): Unilateral preoperative chest wall irradiation in bilateral tissue expander breast reconstruction with acellular dermal matrix: A prospective outcomes analysis. Plast. Reconstr. Surg., 131:921-927.

17. Nguyen MD, Chen $C$, Colakoğlu $S$ et al. (2010): Infectious complications leading to explantation in implant-based breast reconstruction with AlloDerm. Eplasty,10: 404-411.

18. Mentor PM (2006): Important information for reconstruction patients about Mentor MemoryGel silicone gel-filled breast implants. Irving, Texas: Mentor Corp. http://

www.accessdata.fda.gov/cdrh_docs/pdf3/p 030053e.pdf.

19. Allergan Core Study (2006): Important information for women about breast reconstruction with Inamed silicone-filled breast implants. Irvine, Calif: Allergan, Inc., $\quad$ http:// www.accessdata.fda.gov/cdrh_docs/pdf2/P 020056e.pdf.

20. Rice RD, Ayubi FS, Shaub ZJ et al. (2010): Comparison of surgisis, AlloDerm and Vicryl woven mesh grafts for abdominal wall defect repair in an animal model. Aesthetic Plast. Surg.,34: 290-296.

21. Komorowska-Timek E, Oberg KC, Timek TA et al. (2009): The effect of AlloDerm envelopes on periprosthetic capsule formation with and without radiation. Plast. Reconstr. Surg.,123: 807816.

22. Stump A, Holton LH III, Connor J et al. (2009): The use of acellular dermal matrix to prevent capsule formation around implants in a primate model. Plast. Reconstr. Surg., 124:82-91.

23. Basu CB, Leong $M$ and Hicks MJ (2009): Does acellular cadaveric dermis (ACD) affect breast implant capsule formation in reconstructive breast surgery? A histopathologic comparison of breast capsule and ACD. Plast. Reconstr. Surg., 124:62-63. 